\title{
Use of a motion phantom to verify dose accuracy in different delivery techniques for lung tumors in stereotactic body radiation therapy
}

\author{
Yi-Ru Chen ${ }^{1}$, Fang-Yuh Hsu ${ }^{2}$, Cheng-Ching Yu ${ }^{3}$, Chih-Chieh Chang ${ }^{1}$, Hao-Wen Cheng ${ }^{1}$, \\ Wei-Lun Sun ${ }^{1}$, Jo-Ting Tsai ${ }^{1,4}$
}

${ }^{1}$ Department of Radiation Oncology, Shuang Ho Hospital, Taipei Medical University, New Taipei City, Taiwan; ${ }^{2}$ Nuclear Science and Technology Development Center, National Tsing Hua University, Hsinchu City, Taiwan; ${ }^{3}$ Department of Medical Imaging and Radiological Technology, Yuanpei University of Medical Technology, Hsinchu City, Taiwan; ${ }^{4}$ Department of Radiology, School of Medicine, College of Medicine, Taipei Medical University, Taipei, Taiwan

Contributions: (I) Conception and design: CC Chang, WL Sun, YR Chen; (II) Administrative support: FY Hsu, CC Yu, JT Tsai; (III) Provision of study material or patients: JT Tsai, YR Chen; (IV) Collection and assembly of data: CC Chang, YR Chen; (V) Data analysis and interpretation: HW Cheng, CC Chang, YC Chen; (VI) Manuscript writing: All authors; (VII) Final approval of manuscript: All authors.

Correspondence to: Jo-Ting Tsai, MD, PhD. Department of Radiation Oncology, Shuang Ho Hospital, Taipei Medical University, No. 291, Zhongzheng Rd., Zhonghe Dist., New Taipei City 235, Taiwan. Email: kitty4024@gmail.com.

Background: Stereotactic body radiation therapy (SBRT) is the standard treatment for inoperable early-stage non-small cell lung cancer (NSCLC). However, respiration-induced tumor motion results in a nonuniform cumulative dose delivery to a lung tumor. In this study, we used a motion phantom system to investigate the effect of respiration-induced lung tumor displacement on dose delivery in the following three different delivery techniques in SBRT: intensity-modulated radiation therapy (IMRT) SBRT (IMRT-SBRT), volumetric modulated arc therapy (VMAT) SBRT (VMAT-SBRT), and helical tomotherapy (HT) SBRT (HT-SBRT).

Methods: We developed a tumor phantom and used a commercial motion phantom system for dose calculations and measurements at three different respiratory amplitudes (10, 14, and $18 \mathrm{~mm}$ ). Four-dimensional computed tomography (4DCT) was performed to define tumor displacement during breathing for internal target volume (ITV) delineation. IMRT-SBRT, VMAT-SBRT, and HT-SBRT plans were generated for the target with different respiratory amplitudes. A total dose of 60 Gy in 5 fractions was prescribed to the target. An ionization chamber and radiochromic films were used to measure the point dose at the tumor center and the dose profiles, respectively, which were then compared with the calculated doses to verify the accuracy of the dose delivered to the lung tumor.

Results: The HT-SBRT plans showed better conformity index (CI) and homogeneity index (HI) than the IMRT-SBRT and VMAT-SBRT plans for all respiratory amplitudes as follows: the mean CI values were $1.22 \pm 0.10,0.95 \pm 0.02$, and $0.95 \pm 0.02$, and the mean $H I$ values were $1.14 \pm 0.01,1.20 \pm 0.01$, and $1.20 \pm 0.01$ for the HT-SBRT, IMRT-SBRT, and VMAT-SBRT plans, respectively. The results showed that in all three techniques at different respiratory amplitudes, the point dose differences at the tumor center between the calculated and measured doses were all within $\pm 3 \%$ as follows: IMRT-SBRT $=1.7 \% \pm 0.7 \%$, VMAT-SBRT $=1.1 \% \pm 1.4 \%$, and HT-SBRT $=-0.7 \% \pm 0.7 \%$. Moreover, regarding the dose profile measurements at different respiratory amplitudes, all film results showed that an adequate treatment dose coverage was obtained in the lung tumor (100\% isodose curve encompassed the lung tumor).

Conclusions: All SBRT plans generated based on 4DCT images ensured adequate treatment coverage, and the point dose differences between the calculated and measured doses were all within $\pm 3 \%$ despite respiratory motion during beam delivery. The HT-SBRT plans showed better HI, CI, and agreement between the measured and calculated doses than the IMRT-SBRT and VMAT-SBRT plans at different respiratory amplitudes.

$\wedge$ ORCID: 0000-0002-5328-9405. 
Keywords: Motion phantom; stereotactic body radiation therapy (SBRT); intensity-modulated radiation therapy (IMRT); volumetric modulated arc therapy (VMAT); helical tomotherapy (HT)

Received: 16 June 2021; Accepted: 06 September 2021; Published: 30 December 2021.

doi: $10.21037 /$ tro-21-14

View this article at: https://dx.doi.org/10.21037/tro-21-14

\section{Introduction}

According to the Ministry of Health and Welfare of Taiwan, cancer has been the leading cause of death (among the top 10) for many years. In particular, lung cancer was the leading cause of cancer-related deaths (among the top 10) in 2016. Nearly 9,000 patients in Taiwan die of lung cancer each year, and lung cancer is the leading cause of cancerrelated death among both men and women. According to the stereotactic body radiation therapy (SBRT) guidelines from the American Society for Radiation Oncology (ASTRO), SBRT is one of the standard treatments for inoperable early-stage non-small cell lung cancer (NSCLC) with a lesion diameter of $5 \mathrm{~cm}$ or below (1-3).

Goitein $e t a l$. stated that a tumor must be delineated from the surrounding normal tissues or the dose delivered to the peripheral target region may be insufficient. Moreover, tumor motion and treatment techniques may result in a nonuniform dose to the target, highlighting the importance of estimating displacement uncertainties (4).

Guckenberger $e t$ al. investigated the effect of respiratory motion on the SBRT treatment site in lung cancer patients. With pretreatment cone-beam computed tomography (CBCT), the authors found that the average tumor displacement was $2.8 \pm 1.6 \mathrm{~mm}$ and recommended a margin of $5 \mathrm{~mm}$ for the internal target volume (ITV) (5).

Rao et al. compared volumetric modulated arc therapy (VMAT) plans with helical tomotherapy (HT) and step-and-shoot intensity-modulated radiation therapy (IMRT) plans. The results showed that both VMAT and HT plans can provide more uniform target doses and improve normal tissue sparing compared with fixed field IMRT plans. Both VMAT and HT plans can be delivered accurately based on their QA standards. Overall, VMAT was able to provide an approximately $40 \%$ reduction in the treatment time while maintaining the plan quality comparable to that of HT (6).

Ahmed et al. compared NSCLC patients with stage I to IV disease using three-dimensional computed tomography (3DCT) and four-dimensional computed tomography
(4DCT) simulation scans under free breathing, and found that the anatomical position of the planning treatment volume (PTV) depends on the tumor and lymph node movement during the respiratory cycle. Therefore, all lung cancer patients who receive curative radiotherapy are recommended to undergo 4DCT simulation to ensure accurate coverage of the target volume (7).

It is difficult to quantify the actual dose delivered to a tumor due to tumor displacement associated with respiratory motion. Therefore, in this study, we used a motion phantom system to investigate the effect of respiration-induced lung tumor displacement on dose delivery in the following three different techniques: IMRT-SBRT, VMAT-SBRT, and HTSBRT. 4DCT was used to define the treatment area while considering respiratory motion. An ionization chamber and radiochromic films were used to measure the point dose at the tumor center and the dose profiles, respectively, which were then compared with the calculated doses to verify the accuracy of the dose delivered to the lung tumor.

We present the following article in accordance with the MDAR reporting checklist (available at https://dx.doi. org/10.21037/tro-21-14).

\section{Methods}

The study flowchart is shown in Figure 1.

\section{Motion phantom system}

In this study, we used a motion phantom system to verify the calculated dose. This system included a CIRS model 008A dynamic thorax phantom (CIRS Inc., VA, USA), CIRS respiratory motion control, and a homemade dosemeasuring phantom (see Figure 2 for the design process). The CIRS respiratory motion control program was used to select the respiratory amplitude and cycle time, and the homemade dose measuring phantom was driven by different breathing patterns to simulate lung tumor displacement associated with different respiratory amplitudes (Figure 3). 


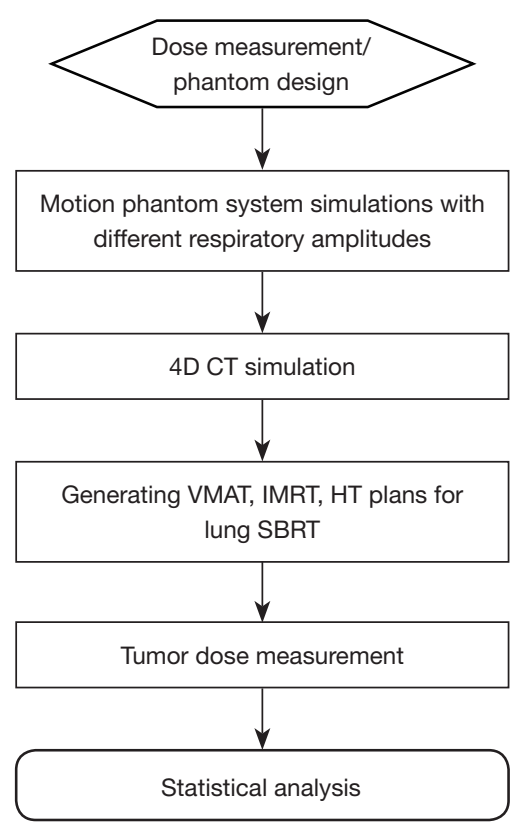

Figure 1 Flowchart of the research design.

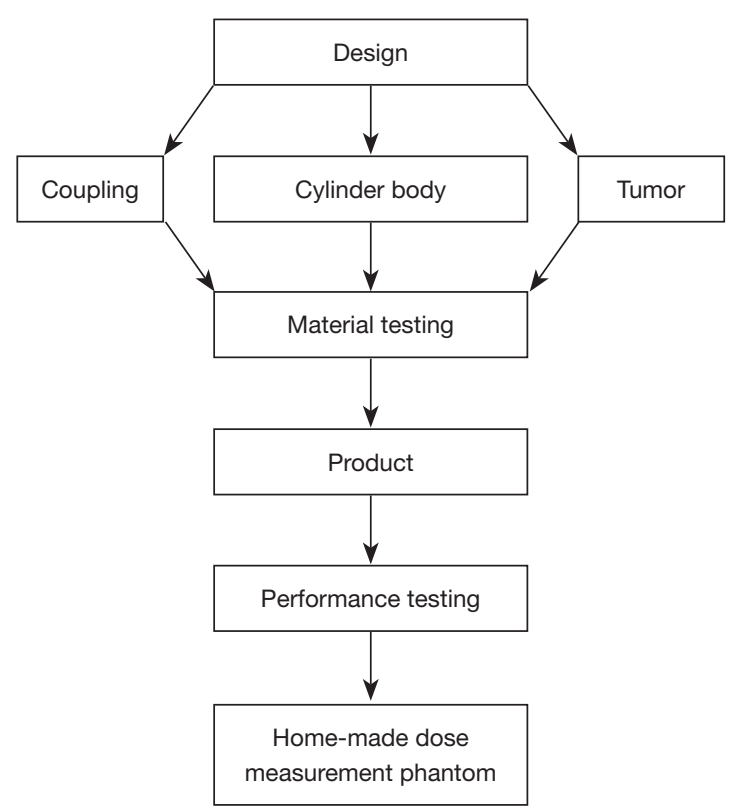

Figure 2 Flowchart of the homemade phantom for the dose measurement.

\section{CIRS thorax phantom and respiratory motion control}

The CIRS thorax phantom represents the shape, proportion, and composition of an average human thorax. The phantom is composed of tissue and lung equivalent
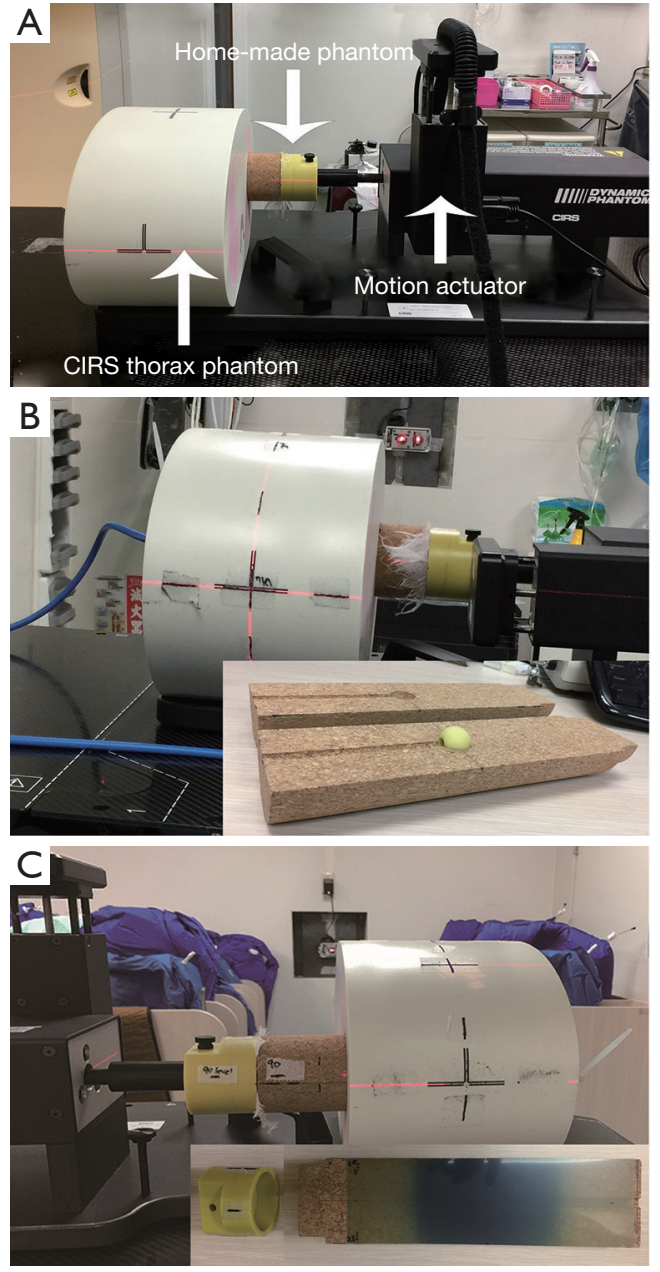

Figure 3 This system included a CIRS respiratory motion control, and a homemade dose-measuring phantom. (A) Motion phantom system. (B) Point dose measurement at the center of the tumor phantom. (C) Dose profile measurement setup established to measure the dose profiles in the $x-y$ and $y-z$ planes.

materials with simplified geometries and contains an anthropomorphic spine with cortex and trabecular bones. The CIRS respiratory motion controller is used to control translational and rotational motions to simulate complex $3 \mathrm{D}$ motions. The motion actuator, which is connected to the phantom and the motion controller, can be driven by the controller (Figure $3 A$ ). In this study, we used the Cos4 waveform to simulate respiratory motion in the inferior-superior (IS), anterior-posterior (AP) and left/ right (LR) directions. Fukumitsu et al. showed that the average respiratory rate was 10 to 18 breaths/min, with approximately 3 to 6 seconds per respiratory cycle and 

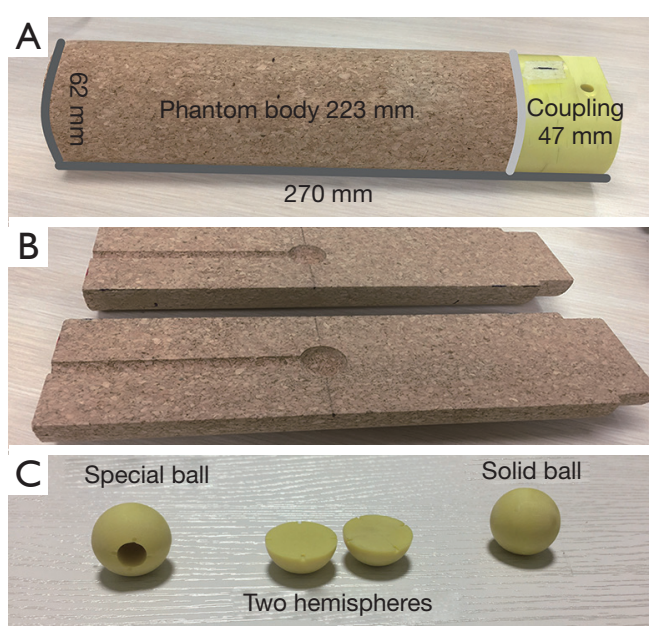

Figure 4 The homemade dose-measuring phantom. (A) Selfmade cylindrical phantom for dose measurements. (B) Cylindrical phantom body composed of two special semicylindrical cylinders. (C) Three spherical tumor inserts.

a respiratory amplitude of 5 to $25 \mathrm{~mm}$ (8). This study was designed to simulate regular respiratory motion during SBRT, with a respiratory rate of 13 breaths/min, a respiratory cycle of 4.5 seconds, and respiratory amplitudes of 10,14 , and $18 \mathrm{~mm}$.

\section{Homemade dose measurement phantom}

The homemade dose-measuring phantom was a column with a height of $270 \mathrm{~mm}$ and a bottom diameter of $62 \mathrm{~mm}$ and was a lung tissue substitute. The phantom included a coupler used to connect the phantom to the motion actuator and a spherical tumor insert. The phantom consisted of two customized semicylinders, with the front end connected to the coupler and a notch $95 \mathrm{~mm}$ from the rear end for the spherical tumor insert (Figure 4). Different types of spherical tumors can be inserted into the phantom body as needed for point dose or dose profile measurements. In this study, a cork with a physical density of $0.27 \mathrm{~g} / \mathrm{cm}^{3}$ was used as the material for the phantom body based on the results reported by Chang et al. as its physical properties (physical density, electron density, and effective atomic number) are very similar to those of lung tissue (9). The material of the coupler was acrylonitrile butadiene styrene (ABS) resin with a density of approximately 1.04 to $1.06 \mathrm{~g} / \mathrm{cm}^{3}$.

Given the use of different types of dosimeters, three spherical tumor inserts with diameter of $20 \mathrm{~mm}$ were designed with distinct functions (Figure 4). (A) A tumor insert with a spherical cavity to accommodate the ionization chamber for the point dose measurements. (B) A tumor insert consisting of two semispherical parts and radiochromic film was placed between the two semispherical parts to measure the dose profile. (C) A solid tumor insert was used to simulate the tumor size and shape. ABS resin with a physical density of $1.02 \mathrm{~g} / \mathrm{cm}^{3}$ was selected as the material for the tumor inserts based on the density of human tissue recommended by Woodard et al. (10).

\section{$4 D C T$}

A Philips Brilliance Big Bore CT scanner (Philips Medical Systems, Cleveland, OH, USA) was used for the 4DCT scans. The bellows device for the breathing monitor was attached to the motion actuator of the CIRS thorax phantom. A 4DCT scan was performed according to the clinical protocol for SBRT (pitch: 0.09, rotation time: 0.5 seconds, $120 \mathrm{kV}$, $350 \mathrm{mAs}$, respiratory rate: 13 breaths/min, axial thickness: $3 \mathrm{~mm}$, helical mode) (11). The 4DCT data were reconstructed into ten phases ( $0 \%$ to $90 \%)$. Maximum intensity projection $\mathrm{CT}$ images $\left(\mathrm{CT}_{\mathrm{MIP}}\right)$ and average $\mathrm{CT}$ images $\left(\mathrm{CT}_{\mathrm{AVG}}\right)$ were also reconstructed from the 4DCT data.

\section{SBRT plans}

The internal target volume (ITV) was generated based on the tumor displacement data from 4DCT. The PTV was defined as the ITV with an isotropic expansion of a 5 -mm margin. A total dose of 6,000 cGy in 5 fractions was prescribed to the PTV. All plans in this study aimed to cover at least $95 \%$ of the PTV by $95 \%$ of the prescribed dose (PD); the minimum dose to the PTV should be higher than $93 \%$ of the $P D$, and the maximum dose $\left(D_{\max }\right)$ should be less than $120 \%$ of the PD.

IMRT-SBRT, VMAT-SBRT, and HT-SBRT plans were generated for the target with different respiratory amplitudes. The HT-SBRT plans were generated in Hi-ART treatment planning system (TPS) using the convolution/superposition algorithm with $1.95 \mathrm{~mm}$ grid size (TomoTherapy Inc., Version 5.1.2, Madison, WI, USA). The VMAT-SBRT and IMRT-SBRT plans with 6-MV photon energy were generated for an Elekta Synergy linear accelerator (linac) (Elekta AB, Stockholm, Sweden) in Pinnacle TPS using the adaptive convolution algorithm with 2 mm grid size (Philips, Version 9.8.0, Fitchburg, WI, USA).

The VMAT-SBRT and IMRT-SBRT plans with 6-MV photon energy were generated for an Elekta Synergy linear 
accelerator (linac) (Elekta AB, Stockholm, Sweden) by using a Pinnacle TPS (Philips, Version 9.8.0, Fitchburg, WI, USA). For the IMRT-SBRT plans, 12 beams with the step-andshoot technique were used for optimization, including ten coplanar beams (gantry angle $=195^{\circ}, 230^{\circ}, 300^{\circ}, 335^{\circ}, 10^{\circ}$, $40^{\circ}, 70^{\circ}, 100^{\circ}, 130^{\circ}$, and $160^{\circ}$ ) and two noncoplanar beams (gantry angles of $20^{\circ}$ and $340^{\circ}$ with couch angle of $70^{\circ}$ ). For the VMAT-SBRT plans, four coplanar partial arcs of $335^{\circ}$ to $180^{\circ}$ in clockwise and counterclockwise directions were used. The VMAT-SBRT plans were optimized with a gantry spacing of $4^{\circ}$, a constraint leaf motion of $0.33 \mathrm{~cm} / \mathrm{deg}$, and a maximum variable dose rate of $600 \mathrm{MU} / \mathrm{min}$. For the HTSBRT plans, a field width of $2.5 \mathrm{~cm}$ and a pitch of 0.217 were used for optimization.

\section{Plan evaluation}

The conformity index (CI) and homogeneity index (HI) of the IMRT-SBRT, VMAT-SBRT, and HT-SBRT plans were calculated at different respiratory amplitudes.

The CI is defined as follows (12):

$$
C I=P I V / P T V
$$

where PIV is the prescription isodose volume, and PTV is the planning target volume. Based on the definition, a CI value closer to 1 corresponds to better conformity. According to the Radiation Therapy Oncology Group (RTOG) recommendation, the normal range of the $\mathrm{CI}$ is 1.0 to 2.0, indicating no deviation from the RTOG protocol. A CI value $>2.0$ or $<1.0$ indicates mild deviations, and a CI value $>2.5$ or $<0.9$ indicates major deviations (12).

The HI is defined as follows (12):

$$
H I=D_{\max } / P D
$$

where $\mathrm{D}_{\text {max }}$ is the maximum dose to the PTV, and PD is the prescribed dose. A HI value closer to 1 corresponds to better homogeneity in the target. According to the RTOG recommendation, the $\mathrm{HI}$ should not exceed 2.0. An $\mathrm{HI}>2.0$ indicates mild deviations, and an $\mathrm{HI}>2.5$ indicates major deviations (12). The beam-on time (BOT) of IMRT- and VMAT-SBRT were measured by a timer excluding the time for couch rotation between fields. As for the BOT of HTSBRT, we obtained from the TPS directly.

\section{Dose measurement}

In this study, an ionization chamber (PTW 31010,
0.125 cc Semiflex Chamber) and EBT3 films were used to measure the point dose at the tumor center and the dose profiles, respectively. The EBT3 films were cut to $20.5 \mathrm{~cm} \times 6.2 \mathrm{~cm}$ and placed in the homemade phantom to measure the dose profiles along the $\mathrm{X}, \mathrm{Y}$, and $\mathrm{Z}$ axes. Previous studies reported that the dose measurement uncertainties in SRS and SBRT using EBT3 films were approximately $1.5 \%(13,14)$.

\section{Point dose measurement at the tumor center}

To minimize variations in the experimental results due to temperature, atmospheric pressure, and device status, prior to each measurement, we used a solid water phantom and an ionization chamber (PTW-31010) to obtain the dose conversion factor of the photon beam $(\mathrm{cGy} / \mathrm{nC})$ based on our clinical condition of $1 \mathrm{cGy} / \mathrm{MU}$ in the solid water phantom. For the point dose measurement, the ionization chamber was placed in the homemade dose-measuring phantom (Figure 3B) to measure the point dose of IMRT, VMAT, and HT at three different respiratory amplitudes. The measurement was repeated three times, and the mean values were calculated and compared with the doses calculated in the treatment plans.

\section{Dose profile measurement}

We established the EBT3 film calibration curve (optical density $v s$. dose) prior to the dose profile measurements. All EBT3 films used for measurements were from the same lot (Lot\#: 06141702). The EBT3 film was sandwiched between the two semispherical inserts positioned inside the cork semicylinders of the homemade phantom. Then, the phantom connected to the coupler was rotated for the dose profile measurements in the $x-y$ and $y-z$ planes for nine treatment plans as follows: three respiratory amplitudes, $18 \mathrm{~mm}, 14 \mathrm{~mm}$, and $10 \mathrm{~mm}$, and three techniques, IMRT-SBRT, VMAT-SBRT, and HTSBRT (Figure 3C). An EPSON 10000 XL Scanner (48-bit RGB) was used to scan the irradiated EBT3 films, which were then imported into Film QA software (Ashland ISP Inc., Covington, KY, USA) along with the TPS-calculated doses for the analysis.

\section{Statistical analysis}

A Mann-Whitney test was used for the statistical analysis of the different SBRT techniques. A P value $\leq 0.05$ was considered statistically significant. 
Table 1 Plan evaluation parameters of the IMRT-, VMAT-, and HT-SBRT plans at different respiratory amplitudes

\begin{tabular}{|c|c|c|c|c|c|}
\hline Variable & Amplitude (mm) & IMRT & VMAT & $\mathrm{HT}$ & $P$ value \\
\hline \multirow{3}{*}{$\mathrm{Cl}$} & 14 & 0.95 & 0.95 & 1.27 & \\
\hline & 10 & 0.93 & 0.93 & 1.11 & \\
\hline & Mean $\pm S D$ & $0.95 \pm 0.02$ & $0.95 \pm 0.02$ & $1.22 \pm 0.10$ & $\mathrm{a},{ }^{*} \mathrm{~b},{ }^{*} \mathrm{c}$ \\
\hline \multirow{3}{*}{$\mathrm{HI}$} & 14 & 1.20 & 1.20 & 1.13 & \\
\hline & 10 & 1.19 & 1.20 & 1.14 & \\
\hline & Mean \pm SD & $1.20 \pm 0.01$ & $1.20 \pm 0.01$ & $1.14 \pm 0.01$ & $\mathrm{a},{ }^{*} \mathrm{~b},{ }^{*} \mathrm{c}$ \\
\hline \multirow[t]{2}{*}{ BOT (sec) } & 18 & 637.0 & 330.0 & 742.1 & \\
\hline & Mean \pm SD & $662.3 \pm 37.2$ & $333.0 \pm 9.8$ & $694.8 \pm 51.8$ & ${ }^{*} \mathrm{a},{ }^{*} \mathrm{~b}, \mathrm{c}$ \\
\hline
\end{tabular}

a, IMRT vs. VMAT; b, VMAT vs. HT; c, HT vs. IMRT. *, P $\leq 0.05$. Cl, conformity index; HI, homogeneity index; BOT, beam on time. IMRT, intensity-modulated radiation therapy; VMAT, volumetric modulated arc therapy; HT-SBRT, helical tomotherapy stereotactic body radiation therapy.
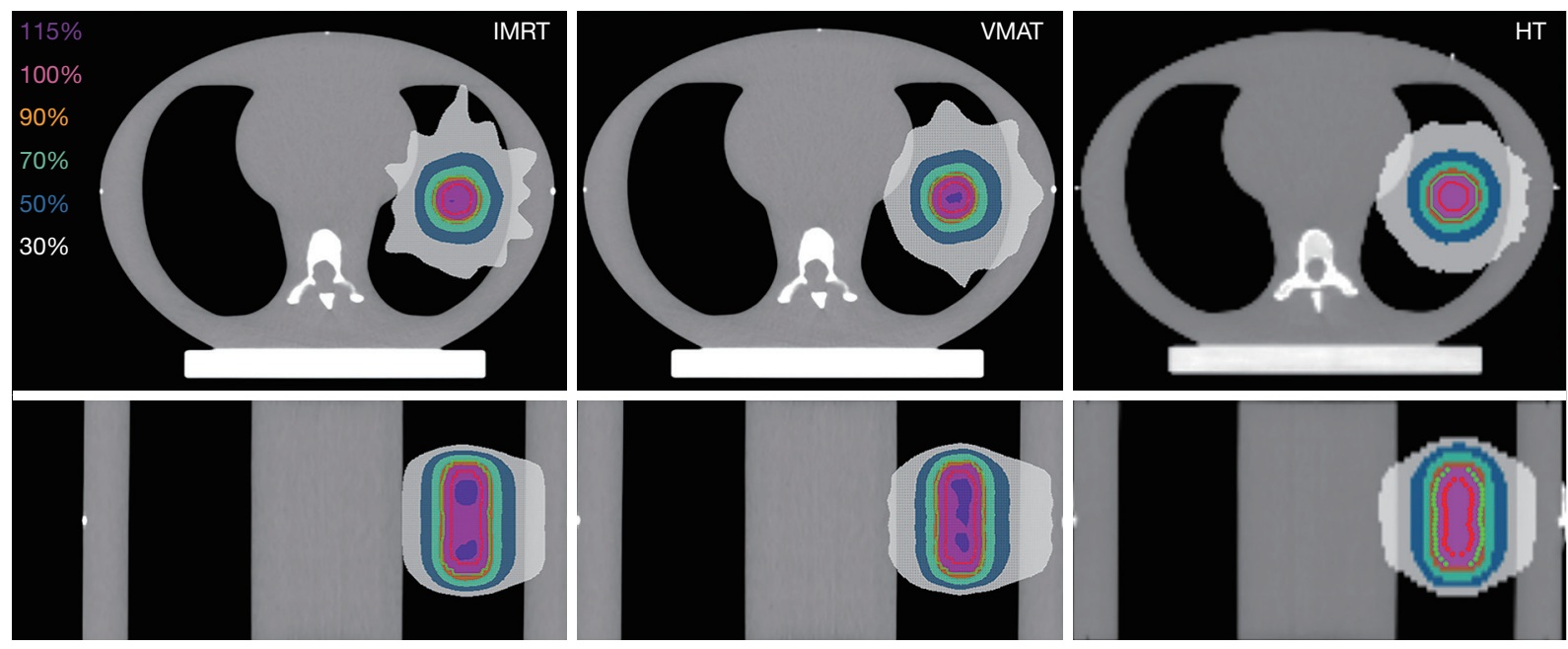

Figure 5 Dose distributions of IMRT-, VMAT, and HT-SBRT plans on axial (top) and coronal (bottom) images at the maximum respiratory amplitude $(18 \mathrm{~mm})$. The green line indicates PTV, and the red line indicates ITV. IMRT, intensity-modulated radiation therapy; VMAT, volumetric modulated arc therapy; HT-SBRT, helical tomotherapy stereotactic body radiation therapy; PTV, planning treatment volume; ITV, internal target volume.

\section{Results}

\section{CI, HI and BOT of different SBRT plans}

The plan evaluation parameters of the different techniques are summarized in Table 1. The dose distributions of different techniques on axial and coronal images at the maximum respiratory amplitude $(18 \mathrm{~mm})$ are shown in Figure 5 .

For HT-SBRT plans, the mean CI at different respiratory amplitudes was $1.22 \pm 0.10$, which was in the normal range (1.0-2.0). For the IMRT-SBRT and VMAT-SBRT plans, the 
Table 2 Point dose differences at the center of the tumor between the calculated, measured and prescribed doses of three techniques at different respiratory amplitudes.

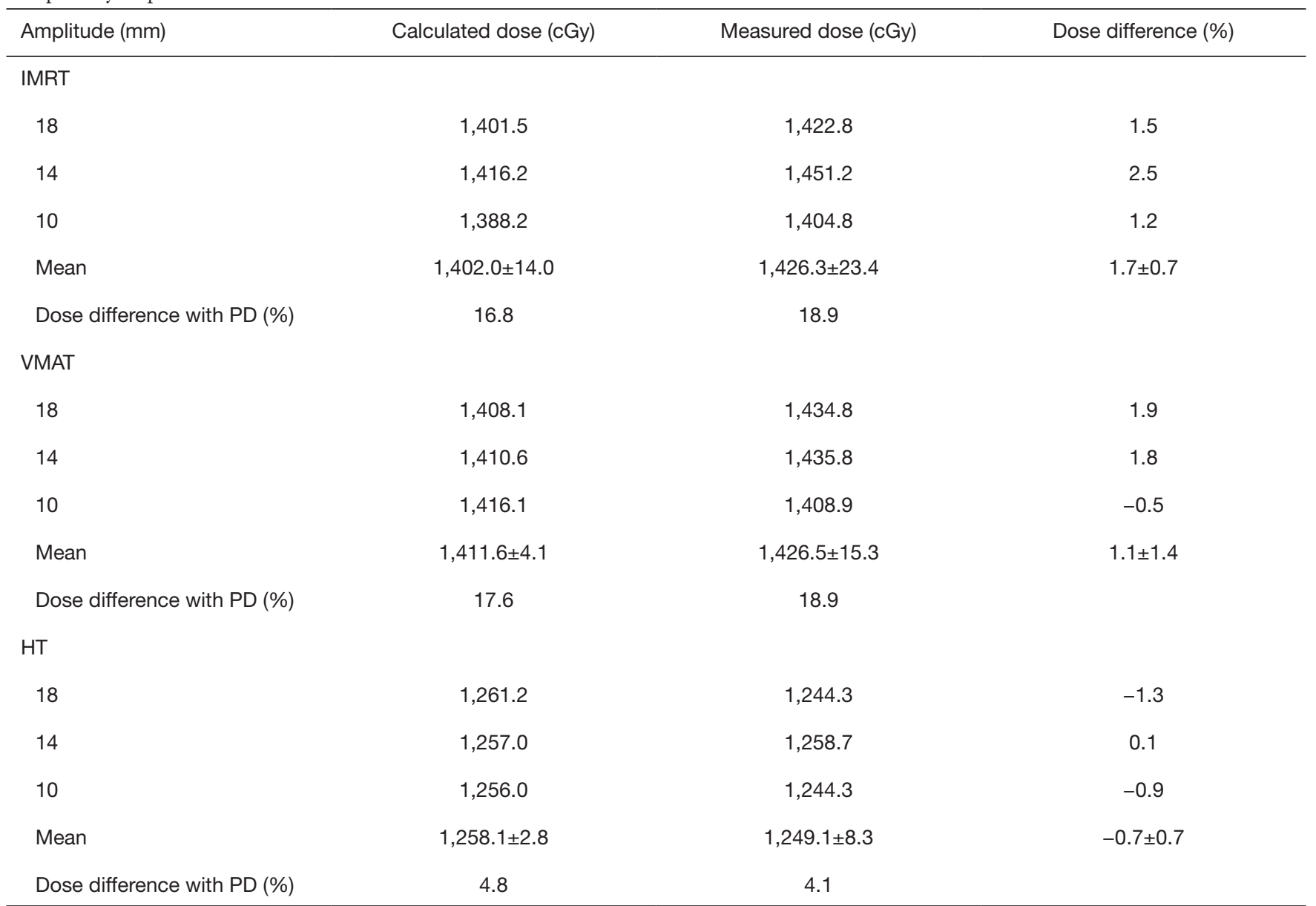

Dose difference $(\%)=[($ average measured dose - calculated dose $) /$ calculated dose $] \times 100 \%$. Dose difference with PD $(\%)=[($ average dose $-1,200) / 1,200] \times 100 \%$. IMRT, intensity-modulated radiation therapy; VMAT, volumetric modulated arc therapy; HT, helical tomotherapy; PD, prescribed dose.

mean CI values at different respiratory amplitudes were both $0.95 \pm 0.02$, indicating mild deviation from the RTOG protocol (>2.0 or $<1.0)$. The HT-SBRT plans showed a statistically significantly better mean CI than the IMRT-SBRT and VMAT-SBRT plans, but there was no statistically significant difference between the IMRT-SBRT and VMAT-SBRT plans. For the HT-SBRT plans, there was a trend toward a better CI with decreasing respiratory amplitude; however, the IMRTSBRT and VMAT-SBRT plans showed the opposite trend. For HI, the mean HI of the HT-SBRT plans with different respiratory amplitudes was $1.14 \pm 0.01$, which was closer to 1 than those of the IMRT-SBRT $(1.20 \pm 0.01)$ and VMAT-SBRT plans $(1.20 \pm 0.01)$ (Table 1). The HT-SBRT plans showed a statistically significantly lower mean HI than the IMRTSBRT and VMAT-SBRT plans, but there was no statistically significant difference between the IMRT-SBRT and VMATSBRT plans. For the HT-SBRT plans, there was also a trend toward a better HI with decreasing respiratory amplitude. The VMAT-SBRT plans showed statistically significantly shorter BOT (average, 333.0 $\pm 9.8 \mathrm{sec}$ ) than the other plans. There was no significant difference in the BOT between the IMRT-SBRT (average, $662.3 \pm 137.2 \mathrm{sec}$ ) and HT-SBRT plans (average, 694.8 $\pm 51.8 \mathrm{sec}$ ).

\section{Point dose measurement at the tumor center}

The results showed that for all three techniques at different respiratory amplitudes, the point dose differences at the tumor center between the calculated and measured doses were all within $\pm 3 \%$ (Table 2): IMRT-SBRT $=1.7 \% \pm 0.7 \%$, 
Table 3 Measured dose profiles along different axes of three SBRT techniques at different respiratory amplitudes (area covered by $100 \%$ isodose curve)

\begin{tabular}{llll}
\hline Amplitude $(\mathrm{mm})$ & X-axis $(\mathrm{mm})(\mathrm{X} 1 / \mathrm{X} 2)$ & Y-axis $(\mathrm{mm})(\mathrm{Y} 1 / \mathrm{Y} 2)$ & Z-axis $(\mathrm{mm})(\mathrm{Z} 1 / \mathrm{Z2})$ \\
\hline IMRT & & & \\
18 & $38.04(17.75 / 20.29)$ & $54.62(23.21 / 31.41)$ & $35.12(16.78 / 18.34)$ \\
14 & $37.06(15.8 / 21.26)$ & $53.65(24.19 / 29.46)$ & $36.09(17.75 / 18.34)$ \\
10 & $37.06(17.75 / 19.31)$ & $49.74(20.28 / 29.46)$ & $37.07(18.73 / 18.34)$ \\
VMAT & $38.33(19.36 / 18.97)$ & $30.92(22.81 / 48.11)$ & $36.88(19.61 / 17.27)$ \\
18 & $33.16(13.85 / 19.31)$ & $56.57(25.16 / 31.41)$ & $31.21^{*}(16.78 / 14.43)$ \\
14 & $34.14(14.83 / 19.31)$ & $52.67(20.28 / 32.39)$ & $34.14(17.75 / 16.39)$ \\
10 & & & $34.23(16.82 / 17.41)$ \\
HT & $31.78^{*}(14.37 / 17.41)$ & $48.89^{*}(23.26 / 25.63)$ & $37.16(17.80 / 19.36)$ \\
18 & $36.18(15.84 / 20.34)$ & $48.89^{*}(20.33 / 28.56)$ & $34.23(16.82 / 17.41)$ \\
10 & $34.22(14.86 / 19.36)$ & $48.89^{*}(20.33 / 28.56)$ & 3 \\
\hline
\end{tabular}

*, indicate the minimum coverages of the X-, Y-, and Z-axes. IMRT, intensity-modulated radiation therapy; VMAT, volumetric modulated arc therapy; HT, helical tomotherapy.

VMAT-SBRT $=1.1 \% \pm 1.4 \%$, and HT-SBRT $=-0.7 \% \pm 0.7 \%$. The IMRT-SBRT and VMAT-SBRT plans showed that the calculated doses were lower than the measured doses at different respiratory amplitudes, except for VMATSBRT at the $10-\mathrm{mm}$ respiratory amplitude. However, for the HT-SBRT plans at different respiratory amplitudes, the calculated doses were all higher than the measured doses. The results showed that for the IMRT-SBRT and VMAT-SBRT plans at different respiratory amplitudes, the calculated and measured doses were higher than the PD (1,200 cGy per fraction) as follows: the mean calculated doses were $17.6 \%$ and $16.8 \%$ higher than the PD for the IMRT-SBRT and VMAT-SBRT plans, respectively; the mean measured doses were both $18.9 \%$ higher than the PD. For the HT-SBRT plans, the mean measured and calculated point dose were respectively $4.8 \%$ and $4.1 \%$ higher than the PD.

\section{Dose profile measurement}

The measured dose profile results are summarized in Table 3. The point dose measured by ionization chamber was used as a dose correction factor to plot the dose profile on the $\mathrm{X}, \mathrm{Y}$, and $\mathrm{Z}$ axes at different amplitudes of the three techniques. The minimum coverages of the $\mathrm{X}-$,, $\mathrm{Y}-$, and Z-axes of the $100 \%$ dose curves of IMRT-SBRT, VMAT-
SBRT, and HT-SBRT were $31.78 \mathrm{~mm}, 48.89 \mathrm{~mm}$, and $31.21 \mathrm{~mm}$, respectively, all of which were larger than the spherical tumor $(20 \mathrm{~mm}$ in diameter). The minimum coverage values are shown in bold in font Table 3. For the three techniques at different respiratory amplitudes, the measured dose profiles along the y-axis were wider than those along the other two axes. Moreover, for IMRT-SBRT and VMAT-SBRT, the width of the dose profile along the $y$-axis increased as the respiratory amplitude increased, and the widest dose profile along the $y$-axis occurred in VMAT-SBRT at the 18-mm respiratory amplitude (Table 3). However, for HT-SBRT, the width of the dose profile along the y-axis remained the same at different respiratory amplitudes. Figure 6 shows the measured dose profiles on the $\mathrm{X}, \mathrm{Y}$, and $\mathrm{Z}$ axes along with the TPS-calculated dose profiles at the maximum respiratory amplitude $(18 \mathrm{~mm})$ for the three SBRT techniques.

\section{Discussion}

In this study, we developed a tumor phantom, which, along with different measuring tools and a commercial thorax phantom, was used to investigate the effect of respirationinduced lung tumor displacement on dose delivery using different SBRT techniques, and three respiratory amplitudes were selected to simulate lung tumor motion. In other 

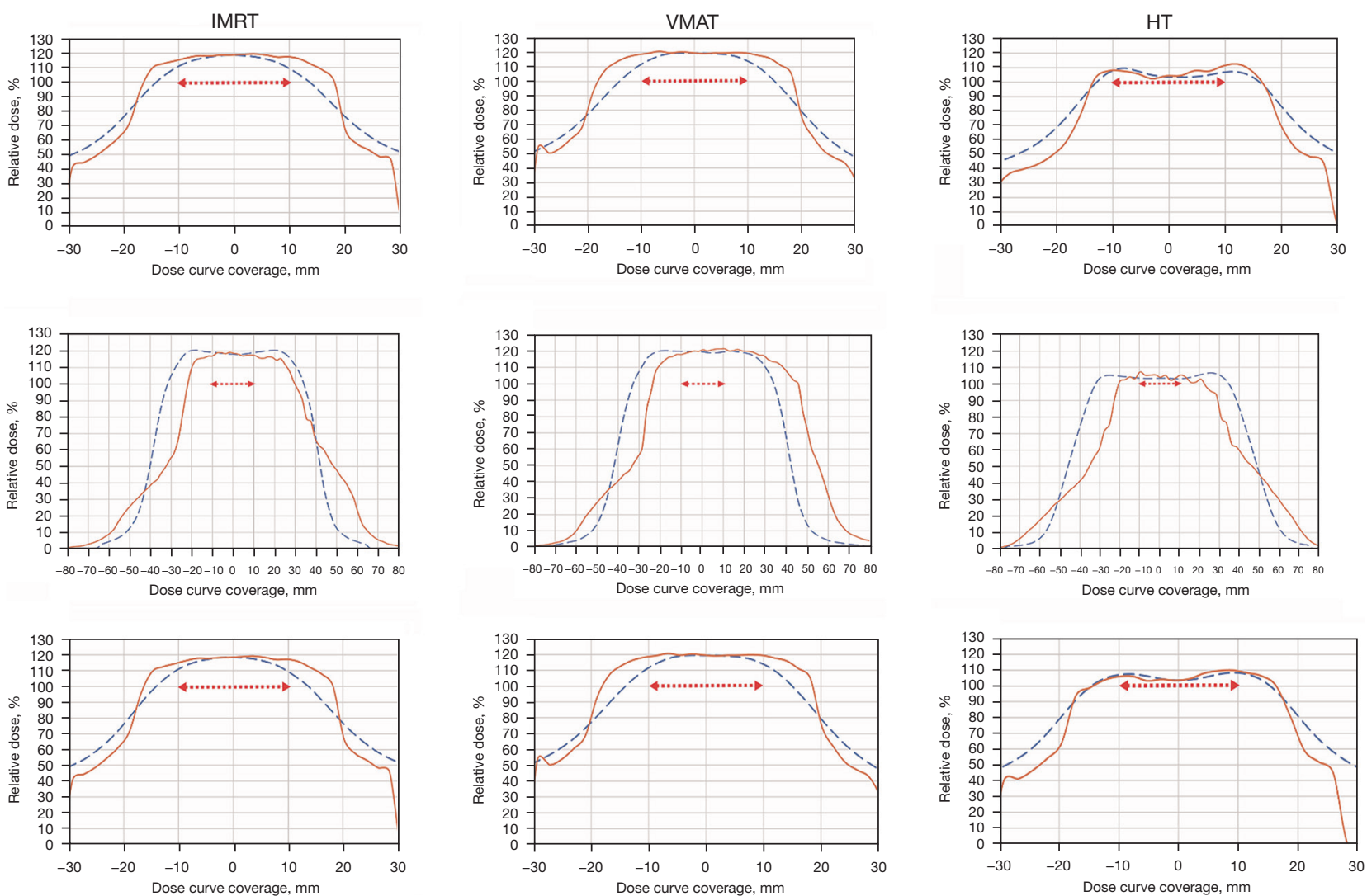

Figure 6 Calculated (blue line) and measured (orange line) dose profiles of three SBRT techniques: (A) X- (B) Y- and (C) Z-axis at the maximum respiratory amplitude $(18 \mathrm{~mm})$. IMRT, intensity-modulated radiation therapy; VMAT, volumetric modulated arc therapy; HT, helical tomotherapy SBRT, stereotactic body radiation therapy.

to represent the dosimetric properties of human lung tissue (average density $0.217 \mathrm{~g} / \mathrm{cm}^{3}$ ), our homemade dosemeasuring phantom was made of cork (density $0.27 \mathrm{~g} / \mathrm{cm}^{3}$ ) instead of commonly used PMMA (density $1.18 \mathrm{~g} / \mathrm{cm}^{3}$ ). Material density is a crucial factor for using phantom to measure lung radiation dose. To take into account tumor motion during beam delivery, 4DCT data were used for ITV delineation. The HT-SBRT plans showed better CI and HI than the IMRT-SBRT and VMAT-SBRT plans.

However, there were no statistically significant differences in CI and HI between the IMRT-SBRT and VMAT-SBRT plans. For CI, the HT-SBRT plans showed a trend toward a better $\mathrm{CI}$ as the respiratory amplitude decreased; however, the IMRT-SBRT and VMAT-SBRT plans showed the opposite trend with a better CI as the respiratory amplitude increased.

For the linac-based SBRT, the width of the multileaf collimator (MLC) could affect the spatial resolution of the dose distribution (15). In this study, a smaller respiratory amplitude indicates a smaller PTV; therefore, due to the MLC with 1-cm-wide leaves at the isocenter for the Synergy linac, our results showed that the CI slightly improved as the respiratory amplitude increased for the linac-based SBRT. For HI, the HI values remained almost the same for the IMRT-SBRT and VMAT-SBRT plans at different respiratory amplitudes, and there was no obvious trend in HI based on the respiratory amplitude with the HT-SBRT plans. The VMAT-SBRT plans with coplanar technique showed approximately $50 \%$ less BOT than the other plans. For the HT-SBRT plans, the BOT decreased as the respiratory amplitude increased. However, for the linac-based SBRT, our results showed a trend toward a shorter BOT as the respiratory amplitude increased. Again, we speculate that the plan complexity would increase for a smaller PTV due to the MLC with wider leaves, thus increasing the BOT.

The HT-SBRT plans showed a smaller difference between the prescribed dose and calculated dose compared 
with the VMAT- and IMRT-SBRT plans. To achieve the planning criteria of SBRT, linac-based SBRT with 1-cmwide MLC leaves could generate SBRT plans with larger high-dose areas (115\%), as shown in Figure 5. Ardu et al. showed that the HT TPS overestimated the measured dose for thorax lesions (16), we optimized the HT-SBRT plans with a minimum PTV dose equal to 103\% PD in this study. Based on the point dose measurement results, we can still observe that the measured doses were slightly lower than the calculated doses at 18- and 10-mm respiratory amplitudes, and the linac-based SBRT plans showed that the measured doses were almost higher than the calculated doses. Therefore, physicians and physicists should be aware of the dose differences at the tumor center between calculated and measured doses for different SBRT techniques. For all three SBRT techniques at different respiratory amplitudes in this study, the point dose differences between the calculated and measured doses were all within $\pm 3 \%$.

For the dose profile measurement, previous studies reported that the largest respiration-induced lung tumor displacement found in the IS direction (17-19). Therefore, we can expect that the measured dose profiles along the $y$-axis would be wider than those along the other two axes at different respiratory amplitudes, which is consistent with the findings of this study (Table 3). The HT-SBRT plans showed that continuous couch movements during beam delivery along with the respiratory movement axis led to narrower $y$-axis dose profiles compared with the IMRTand VMAT-SBRT plans at different respiratory amplitudes. Due to the coplanar VMAT-SBRT technique used in this study, which the beam direction was perpendicular to the IS direction, the VMAT-SBRT plans showed the widest $\mathrm{y}$-axis dose profiles at different respiratory amplitudes. For the IMRT-SBRT plans with two noncoplanar beams, all $y$-axis dose profiles were smaller compared with those of the VMAT-SBRT plans. For the dose profile measurements at different respiratory amplitudes, all film results showed that an adequate treatment dose was obtained in the lung tumor (100\% isodose curve encompassed the lung tumor).

Rao et al. investigated lung SBRT treatment plans generated based on 4D CT images and found a minimal effect of respiratory motion on the tumor dose and minimal dose heterogeneity for both IMRT- and VMAT-SBRT. Weyh et al. (20) analyzed the dosimetric accuracy of lung SBRT using three different techniques, i.e., RapidArc, HT, and IMRT; the results showed that the mean gamma passing rate was $>97 \%$, suggesting good agreement between the measured and calculated doses. These results are consistent with the findings of this study.

This study was designed to simulate regular respiratory motion with different respiratory amplitudes, which was the limitation of the study. Due to the limited range of movement and area of dose measurement of the thorax phantom, we only performed dose analysis in lung tumors with a respiratory amplitude $\leq 18 \mathrm{~mm}$, and did not analyze the dose in the surrounding normal tissues or critical organs. In the future, we aim to investigate the effect of irregular respiratory motions with different respiratory amplitudes on dose delivery in tumors with different volumes for lung SBRT.

In summary, all SBRT plans generated based on 4DCT images ensured adequate treatment coverage, and the point dose differences between the calculated and measured doses were all within $\pm 3 \%$ despite respiratory motion during beam delivery. The HT-SBRT plans showed better HI, CI, and agreement between the measured and calculated doses than the IMRT-SBRT and VMAT-SBRT plans at different respiratory amplitudes. For the linac-based SBRT, our results showed comparable HI and CI for both IMRT- and VMAT-SBR plans. Therefore, VMAT-SBRT with shorter BOT can be a feasible option for patients who cannot tolerate a long radiation treatment time.

\section{Acknowledgments}

This study was supported by the Department of Radiation Oncology, Shuang Ho Hospital. This manuscript was edited by Wallace Academic Editing.

Funding: None.

\section{Footnote}

Reporting Checklist: The authors have completed the MDAR reporting checklist. Available at https://dx.doi. org/10.21037/tro-21-14

Conflicts of Interest: All authors have completed the ICMJE uniform disclosure form (available at https://dx.doi. org/10.21037/tro-21-14). The authors have no conflicts of interest to declare.

Ethical Statement: The authors are accountable for all aspects of the work in ensuring that questions related to the accuracy or integrity of any part of the work are appropriately investigated and resolved.

Open Access Statement: This is an Open Access article 
distributed in accordance with the Creative Commons Attribution-NonCommercial-NoDerivs 4.0 International License (CC BY-NC-ND 4.0), which permits the noncommercial replication and distribution of the article with the strict proviso that no changes or edits are made and the original work is properly cited (including links to both the formal publication through the relevant DOI and the license). See: https://creativecommons.org/licenses/by-nc-nd/4.0/.

\section{References}

1. Baumann P, Nyman J, Lax I, et al. Factors important for efficacy of stereotactic body radiotherapy of medically inoperable stage I lung cancer. A retrospective analysis of patients treated in the Nordic countries. Acta Oncol 2006;45:787-95.

2. Baumann $\mathrm{P}, \mathrm{Nyman} \mathrm{J}$, Hoyer $\mathrm{M}$, et al. Outcome in a prospective phase II trial of medically inoperable stage I non-small-cell lung cancer patients treated with stereotactic body radiotherapy. J Clin Oncol 2009;27:3290-6.

3. Fakiris AJ, McGarry RC, Yiannoutsos CT, et al. Stereotactic body radiation therapy for early-stage non-small-cell lung carcinoma: four-year results of a prospective phase II study. Int J Radiat Oncol Biol Phys 2009;75:677-82.

4. Goitein M. Organ and tumor motion: an overview. Semin Radiat Oncol 2004;14:2-9.

5. Guckenberger M, Meyer J, Wilbert J, et al. Intra-fractional uncertainties in cone-beam CT based image-guided radiotherapy (IGRT) of pulmonary tumors. Radiother Oncol 2007;83:57-64.

6. Rao M, Yang W, Chen F, et al. Comparison of Elekta VMAT with helical tomotherapy and fixed field IMRT: plan quality, delivery efficiency and accuracy. Med Phys 2010;37:1350-9.

7. Ahmed N, Venkataraman S, Johnson K, et al. Does Motion Assessment With 4-Dimensional Computed Tomographic Imaging for Non-Small Cell Lung Cancer Radiotherapy Improve Target Volume Coverage? Clin Med Insights Oncol 2017;11:1179554917698461.

8. Fukumitsu N, Ishida M, Terunuma T, et al. Reproducibility of image quality for moving objects using respiratory-gated computed tomography: a study using a phantom model. J Radiat Res 2012;53:945-53.

9. Chang KP, Hung SH, Chie YH, et al. A comparison of physical and dosimetric properties of lung substitute materials. Med Phys 2012;39:2013-20.

10. Woodard HQ, White DR. The composition of body tissues. Br J Radiol 1986;59:1209-18.

11. Koninklijke Philips Electronics N.V. Respiratory motion management for CT. www.philips.com/BigBoreCT. Philips Healthcare is part of Royal Philips Electronics; 2013.

12. Shaw E, Kline R, Gillin M, et al. Radiation Therapy Oncology Group: radiosurgery quality assurance guidelines. Int J Radiat Oncol Biol Phys 1993;27:1231-9.

13. Sorriaux J, Kacperek A, Rossomme S, et al. Evaluation of Gafchromic ${ }^{\circledR}$ EBT3 films characteristics in therapy photon, electron and proton beams. Phys Med 2013;29:599-606.

14. Wen N, Lu S, Kim J, et al. Precise film dosimetry for stereotactic radiosurgery and stereotactic body radiotherapy quality assurance using Gafchromic ${ }^{\mathrm{TM}}$ EBT3 films. Radiat Oncol 2016;11:132.

15. Huq MS, Das IJ, Steinberg T, et al. A dosimetric comparison of various multileaf collimators. Phys Med Biol 2002;47:N159-70.

16. Ardu V, Broggi S, Cattaneo GM, et al. Dosimetric accuracy of tomotherapy dose calculation in thorax lesions. Radiat Oncol 2011;6:14.

17. Liu HH, Balter P, Tutt T, et al. Assessing respirationinduced tumor motion and internal target volume using four-dimensional computed tomography for radiotherapy of lung cancer. Int J Radiat Oncol Biol Phys 2007;68:531-40.

18. Suh Y, Dieterich S, Cho B, et al. An analysis of thoracic and abdominal tumour motion for stereotactic body radiotherapy patients. Phys Med Biol 2008;53:3623-40.

19. Sarudis S, Karlsson Hauer A, Nyman J, et al. Systematic evaluation of lung tumor motion using four-dimensional computed tomography. Acta Oncol 2017;56:525-30.

20. Weyh A, Konski A, Nalichowski A, et al. Lung SBRT: dosimetric and delivery comparison of RapidArc, TomoTherapy, and IMR.J Appl Clin Med Phys 2013;14:4065.

doi: $10.21037 /$ tro-21-14

Cite this article as: Chen YR, Hsu FY, Yu CC, Chang CC, Cheng HW, Sun WL, Tsai JT. Use of a motion phantom to verify dose accuracy in different delivery techniques for lung tumors in stereotactic body radiation therapy. Ther Radiol Oncol 2021;5:20. 